\title{
ESTUDIO DE LAS NECESIDADES DE INFORMACIÓN EN LAS PYMES DE TUNDAMA Y SUGAMUXI
}

\author{
STUDY OF INFORMATION NEEDS IN THE SMES \\ OF TUNDAMA AND SUGAMUXI
}

\author{
NEGÓCIOS NECESSIDADES DE INFORMAÇÃO \\ ESTUDO NAS PME TUNDAMA E SUGAMUXI
}

\author{
Por: RODRÍGUEZ DÍAZ - Miryam Teresa, GONZÁLEZ MILLÁN - José Javier, \\ GONZÁLEZ M. - Oscar Ulises
}

\begin{abstract}
Estudiante de Doctorado en Administración, Universidad Autónoma de Querétaro. Profesora auxiliar de la Escuela de Administración de empresas, Universidad Pedagógica y Tecnológica de Colombia. Investigadora Grupo de Investigación Management. E-mail: miryamteresa.rodriguez@ uptc.ed.co, Colombia.

Estudiante de Doctorado en Administración, Universidad Autónoma de Querétaro. Profesor asistente de la Escuela de Administración de Empresas, Universidad Pedagógica y Tecnológica de Colombia. Coordinador Grupo de Investigación Management. E-mail: javier.gonzaleamillan@ uptc.edu.co, Colombia.

Magíster en Salud y seguridad en el trabajo, Universidad Nacional de Colombia. Profesor auxiliar de la Escuela de Ingeniería de Minas, Universidad Pedagógica y Tecnológica de Colombia. Investigador Grupo Investigación GEAM. E-mail: oscar.gonzales02@UPTC.edu.co, Colombia.
\end{abstract}

Recibido: 15 de julio de 2015

Aprobación definitiva: 15 de diciembre de 2015

\section{RESUMEN}

La información ocupa un sitial muy importante en el mundo moderno debido a los flujos que constantemente genera, a la amplia capacidad de los motores de búsqueda y de las bases de datos, y a las diversas formas que ofrecen las Tecnologías de la Información y la Comunicación (TIC). Estos mecanismos permiten almacenar, proteger, gestionar, transferir, utilizar y transformar la información en conocimiento útil que puede ser utilizado con diversos fines, dentro de los cuales se encuentran los empresariales. El presente artículo tiene como objeto determinar las diversas necesidades de información en las pymes de los valles de Sugamuxi y Tundama en el Departamento de Boyacá. Para lograrlo, se encuestaron 266 gerentes de estas empresas (173 de Sugamuxi y 93 de Tundama), se utilizó un tipo y método de estudio descriptivo-explicativo, se implementó una técnica estadística de análisis descriptivo, y se aplicó una encuesta estructurada como instrumento de recolección de datos. Dentro de los resultados, 
se identificó que las principales necesidades de información de las pymes son: estudios de mercados, conocimientos de manuales, normas, procedimientos y operación de equipos.

Palabras clave: información, pymes, necesidades, tecnologías, TIC.

JEL: Q2, D6, O3

\begin{abstract}
Information plays a very important role in the modern world due to the flows it constantly generates, to the ample search engines and databases, and to the various forms offered by information and communications technology (ICT). These mechanisms allow storage, protection, management, transfer, use and transformation of information into useful knowledge that can be used for various purposes, i.g., business. This article aims to identify the various information needs of SMEs of Tundama and Sugamuxi valleys in the Department of Boyaca. To achieve this goal, 266 managers of these companies (173 in Tundama and 93 in Sugamuxi) were surveyed, a descriptive and explanatory method of study was used, a statistical technique of descriptive analysis was adopted and a structured survey was used as a tool for data collection. Among the results, the following main information needs of SMEs were identified: market research, knowledge of manuals, standards, procedures and equipment operation.
\end{abstract}

Keywords: Information, SMEs, needs, technologies, ICTs.

JEL: Q2, D6, O3

\title{
RESUMO
}

No mundo moderno, a informação ocupa um lugar muito importante, porque os fluxos gerados constantemente, as grandes motores de busca, bases de dados e capacidade de as várias formas através da informação e tecnologias da comunicação (TIC) que são oferecidos, como tal, estes permitem armazenar, proteger, gerenciar, transferência, uso e transformar a informação em útil para ser usado para várias finalidades, entre as quais o conhecimento do negócio. Assim, o objetivo deste artigo é determinar as diversas necessidades de informação das PME nos vales do Sugamuxi e Tundama no Departamento de Boyaca, para o efeito 266 gestores destas empresas (173 e 93 foram pesquisados Sugamuxi Tundama). O tipo e método de estudo foi descritivo - explicativo; a proposta era descritivo técnica de análise estatística, e no que diz respeito ao inquérito estruturado instrumento de coleta foi utilizado. Entre os resultados, identificouse que as necessidades de informação das PME mais utilizados são: pesquisa de mercado, o conhecimento de manuais, normas, procedimentos e operação do equipamento.

Palavras-chave: informação, requisitos, tecnologias de comunicação, as PME.

JEL: Q2, D6, O3 


\section{INTRODUCCIÓN}

En medio de una sociedad globalizada, la información se convierte en una ventaja que abarca diversos ámbitos, desde lo académico, lo cultural, y lo social, hasta lo empresarial. Es notable que el cambio y la creciente necesidad de estar continuamente actualizados conlleva la exigencia de una mejor y más rápida información en todos los sectores. Por tal motivo, es preciso resaltar la gran importancia que tiene dicha información y las tecnologías que se utilizan para procesarla y enviarla a su destinatario. De ahí que el presente documento tenga como objetivo demostrar porqué se requiere investigar el tema de las necesidades de la información y, en cierta medida, de las tecnologías que la manejan, i.e., las Tecnologías de la Información y la Comunicación (TIC).

La temática de las necesidades de información lleva consigo el concepto de satisfacción, puesto que ésta se resume en "el hecho de dar solución a una duda o a una dificultad, aquietar y convencerse con una razón eficaz, la duda o la queja que se habían formulado" (Calva, 2009, p.2). De acuerdo con esto, el hecho de tener información conduce a sentirnos satisfechos y nos genera cierta tranquilidad y alegría.

Se ha hecho evidente que las Tecnologías de la Información y la Comunicación han reformado de manera sustancial el trabajo, la educación, la economía y los demás ambientes socioeconómicos del mundo. En consecuencia, las TIC se han convertido en un mecanismo valioso para hacer más eficaces y eficientes las labores empresariales, pues permiten un avance formidable en términos de la comunicación organizacional y la gestión empresarial (Rojas, 2004). Adicionalmente, las TIC sirven como elemento diferenciador de marketing corporativo de las empresas modernas; es claro entonces que la información permite a las compañías mejoras sustanciales en cantidad, calidad y precios.

En tal sentido, es casi imposible desconocer en la sociedad moderna, el papel que desempeña la información en las organizaciones del presente siglo, pues el cambio tecnológico es una constante en el mundo de hoy. Lo anterior ha hecho incursionar a las comunidades actuales en nuevas tecnologías, nuevos sistemas y nuevas tendencias afianzadas desde la globalización, en lo que hoy se ha denominado "la sociedad de la información”. Este fenómeno le ha presentado al mundo nuevas oportunidades y retos de avanzada en cuanto a la visión empresarial de la gerencia moderna, logrando que la gran mayoría de los casos se convierta en plataforma estratégica o en ventaja competitiva de las empresas.

Es necesario en esta instancia, acotar que la información, sea cual sea su fin, actualmente se utiliza indistintamente; en contraste, lo que sí está bien definido, tal como lo expone Vilar (s.f., p.1), es que hay una tendencia que concibe al conocimiento y a las TIC como los principales elementos de desarrollo económico de la nueva centuria. Aunque esto puede ser objeto de debate, existe un completo acuerdo en que las futuras necesidades de negocio y las ventajas competitivas estarán soportadas por el uso intensivo de la información a través de las tecnologías de la información y la comunicación. No obstante, tal afirmación podría verse como temeraria en el plano económico, pero es claro que como actividades de soporte a la gestión y a la economía misma, dichas tecnologías definitivamente juegan un 
papel crucial para las organizaciones. Ahora bien, aquellas entidades que desatiendan y no procuren el uso de las TIC, tenderán a perder su ventaja competitiva e incluso comparativa, siendo relegadas en un mercado cambiante y globalizado (Mulet y Pupo, 2009).

Este documento presenta los resultados de una investigación de campo aplicada a las Pymes de los valles de Sugamuxi y Tundama en el Departamento de Boyacá, relacionados con el establecimiento de las necesidades de información empresarial. Para el desarrollo de esta investigación, en primera instancia se averiguó acerca de la distribución económica de las empresas en mención, para luego conocer los grados de información requeridos, aplicando un total de 266 encuestas estructuradas (distribuidas de manera aleatoria entre los gerentes o administradores). El presente documento consta de cuatro capítulos, distribuidos así: en primer lugar se realiza una revisión bibliográfica sobre las necesidades de información y las TIC; después, se presenta el marco metodológico aplicado en la investigación; posteriormente, se referieren los resultados más relevantes del estudio, centrados en presentar los niveles de necesidades de información a nivel corporativo de las empresas en mención; finalmente, se presentan las conclusiones más relevantes derivadas de la investigación.

\section{MARCO TEÓRICO}

\subsection{LAS NECESIDADES DE INFORMACIÓN}

La necesidad de la información es un tema de gran interés, ya que gracias a ella se investiga, se conoce, y se alimenta la mente sobre todo cuanto se quiere profundizar en cualquier área de la vida. El análisis perteneciente a esta temática tiene mucho que ver con la satisfacción, y cómo ésta repercute de forma positiva o negativa en el ser humano.

Es válido afirmar que, aunque es un tema poco común, la necesidad de la información es una característica representativa de las necesidades humanas en su más alto nivel y surge de las personas cuando se requiere un determinado conocimiento. Se presenta muy a menudo cuando los seres humanos reciben un estímulo que da como resultado la necesidad de la información en busca de su satisfacción.

Por tal motivo, las necesidades de información abarcan otras dimensiones como la empresarial, en donde es muy normal encontrar solo mecanismos de transferencia de información (TIC, motores de búsqueda, plataformas, buscadores, etc.), dejando de lado la esencia, que es la necesidad o los tipos de información que se requieren, máxime cuando se debe empezar por el levantamiento de necesidades y el diagnóstico de las mismas.

Si se identifican claramente las necesidades de información al interior de la organización se estarán maximizando los recursos de la empresa. El concepto mismo, según Reitz, citado por Medina (2012: 2), "la necesidad de la información es una brecha que existe en el conocimiento de una persona que se experimenta 
en el nivel consciente como una pregunta, dando lugar a una búsqueda de una respuesta, puede llevarse a cabo con la diligencia cumplida”.

Dentro del mismo criterio, Calva (2009: 2-3) considera que las necesidades de información son un elemento equilibrador que posee un individuo con el ambiente externo, con base en la carencia de conocimiento e información que se tiene en torno a algún hecho, fenómeno o acontecimiento. Igualmente el autor pone de manifiesto que para que un sujeto se sienta satisfecho con respecto a la información, es importante que revise documentos, lo cual constituye la satisfacción material, de igual forma el individuo conseguirá cierta satisfacción emocional cuando se dé cuenta de lo que realmente sucedió al transcurrir el tiempo, es decir, según Calva (2009: 2-3) para alcanzar a plenitud la satisfacción de las necesidades de información se tienen que tomar en cuenta los siguientes factores:

- Con respecto a la información: Disponibilidad, costo, calidad y accesibilidad.

- Con respecto al usuario: Madurez y experiencia del usuario en el uso de los recursos y fuentes informativos, especialización del usuario en el tema donde se enmarcan sus necesidades de información, conocimiento de las fuentes y recursos, idioma e impresiones subjetivas que posea el sujeto.

Teniendo en cuenta lo anterior es necesario reconocer cuatro elementos muy valiosos que permiten hacer una evaluación de la satisfacción en cuanto a las necesidades de información.

\subsubsection{Elementos de la satisfacción}

Calva (2009: 15) presenta lo que ha denominado los cuatro elementos que corresponden a la satisfacción del ser humano en temas de información, clasificándolos en dos grandes grupos, a saber:

1. La pertinencia y la relevancia: tienen que ver con la información que se proporciona a través de algún documento que dé respuesta a lo que se está buscando. También se relacionan con la calidad misma, pues es muy común encontrar informaciones que no poseen la veracidad o la cientificidad requerida.

2. La precisión y la compilación o recopilación: tienen que ver con la búsqueda de información y pueden estar directamente relacionados con el comportamiento informativo, apuntando a la capacidad de filtración para focalizar exactamente lo requerido por los usuarios de la misma.

Por otro lado, dichos elementos (Calva, 2009: 16) obedecen a una serie de pasos del proceso mismo de necesidades de información, que inician con el surgimiento de una necesidad a base de un estímulo, seguido de la manifestación de las necesidades, la cual se pone de manifiesto en un comportamiento informativo, para concluir con la etapa de satisfacción de la necesidad, la cual podría ser positiva o negativa, ante lo cual el proceso tomaría un comportamiento cíclico, hasta satisfacer dicha necesidad informativa. 
Figura $\mathrm{N}^{\circ} 1$. Fases y Elementos de la satisfacción de necesidades de información.

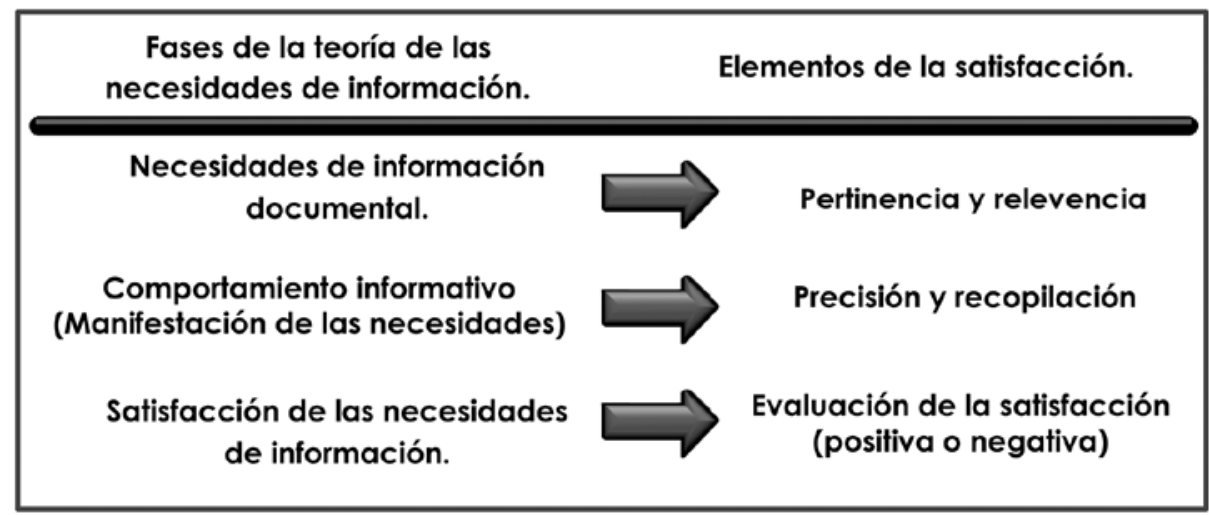

Fuente: Calva, 2009, p. 16

Otro tipo de elementos que conforman la satisfacción de forma positiva o negativa son:

1. Duración: considerado uno de los elementos más importantes en el proceso, debido a que los individuos buscan satisfacer de manera casi inmediata sus requerimientos informativos, para alcanzar plena satisfacción.

2. Superación de la necesidad: se presenta cuando el sujeto se manifiesta a través de algún comportamiento bien sea positivo o negativo que, por lo general, se nota a través de un estado emocional y material, debido al alcance o no de su objetivo.

3. Volumen de Información: se establece en la medida del flujo de información requerida, pues se encontrarán eventos de grandes cantidades de información y otros de la mínima posible.

4. Calidad de la Información: se refiere al grado de complejidad y de asertividad en lo buena o mala que sea la información solicitada por el usuario.

5. Fin de la información: tiene que ver con el uso bueno o malo que se le dé a dicha información y depende del usuario sujeto, ya que puede ser para simplemente adquirir un conocimiento o para utilizarla de manera práctica al interior de la organización.

Según todo lo anterior, la información es cada vez más necesaria en la vida del ser humano; la globalización ha hecho que las personas se interesen de manera más recurrente en ciertos temas, quieran conocer mejores innovaciones en todos los campos y en poco tiempo. Por ello, se han creado a partir del siglo XX y comienzos del XXI las tecnologías de la información y las comunicaciones (TIC) que, aunque no son el objeto principal de este documento, sí se consideran una herramienta de apoyo y transferencia de información y, por tanto, a continuación se considera un apartado de la misma para su análisis (Severin, 2014). 


\subsection{LAS TIC Y LA TRANSFERENCIA TECNOLÓGICA}

\subsubsection{Las Tecnologías de la Información y la Comunicación - TIC}

Coll, Mauri y Onrubia (2008) estipulan que las TIC se refieren a un mecanismo de representación y comunicación novedoso, cuyo uso tiene que ver con sistemas tales como los lenguajes visuales, orales y escritos destinados al tratamiento, transmisión, acceso y uso de la información que se genera al interior de las organizacionales (con o sin ánimo de lucro). En relación al tema, Soler (2008: 1) afirma que las TIC se "refieren a la utilización de múltiples medios tecnológicos o informáticos para almacenar, procesar y difundir todo tipo de información, visual, digital o de otro tipo con diferentes finalidades, como forma de gestionar, organizar en el mundo laboral". En consecuencia, puede decirse que las TIC son herramientas valiosas para fines administrativos, académicos (Foguet, y Donés, 2011) y laborales, por ende, facilitan o diferencian la actividad productiva.

Es importante acotar que, en cuanto a la visión económica es Marx (1946) quien concibe en primer lugar que los mecanismos de trabajo son los que caracterizan dicha actividad, pues en la medida en la que cambian, se van identificando las distintas épocas económicas. Por tanto, los diferentes procesos de tecnificación están relacionados con el uso de herramientas, técnicas, tecnologías y máquinas que sirven para el desarrollo de determinados procesos en la industria (Henao, 2006). Por otra parte, el proceso de trabajo es aquél mediante el cual el hombre entra en contacto con la naturaleza transformándola y transformándose a sí mismo, por medio de la interacción con los diversos elementos que lo componen (Losada, 2010), dentro de los cuales se encuentran: Los medios de trabajo que comprenden las máquinas, herramientas, tecnología, equipos e instalaciones.

Rosario (2006: 2) define las TIC como el "conjunto de tecnologías que permiten la adquisición, producción, almacenamiento, tratamiento, comunicación, registro y presentación de informaciones, en forma de voz, imágenes y datos contenidos en señales de naturaleza acústica, óptica o electromagnética. Las TIC incluyen la electrónica como tecnología base que soporta el desarrollo de las telecomunicaciones, la informática y el audiovisual”. Por su parte, Maldonado, Martínez, García, Aguilera, y González (2010: 57), consideran que "los países en vías de desarrollo deben avanzar en la capacidad y el conocimiento de la economía y la creación para un ambiente favorable para la rápida adopción de las nuevas ideas y de las TIC como una nueva oportunidad de negocios. En particular son las micro, pequeñas y medianas empresas, las que mejor pueden aprovechar las oportunidades que genera un nuevo ambiente de negocios, ya que se pueden beneficiar al momento de integrar las TIC a sus estrategias”. Consecuentemente, queda claro que las TIC se configuran como un factor de impulso a la economía internacional, nacional, regional o local, puesto que la transferencia de información o de conocimiento hace parte del quehacer empresarial y gracias a éstas todo el proceso se hace mucho más fácil y rápido para poner las empresas en el mundo globalizado.

Igualmente, Bonilla y Cubillos, citados por Baelo y Cantón (2009), definen las TIC como "un conjunto de tecnologías desarrolladas para gestionar información y enviarla de un lugar a otro. Comprenden un abanico de soluciones muy extenso", (p. 1). Este concepto permite inferir, que las tecnologías son un apoyo vital para almacenar información y recuperarla después, para enviar y recibir cualquier tipo 
de información y para realizar informes o calcular resultados. Por su parte, Cabero (1998: 1) considera que las nuevas tecnologías de la información y la comunicación son "las que giran en torno a tres medios básicos: la informática, la microelectrónica y las telecomunicaciones; pero giran, no sólo de forma aislada, sino lo que es más significativo de manera interactiva e interconexionadas, lo que permite conseguir nuevas realidades comunicativas".

Al respecto, Pérez y Fernández citados Salguero (2009: 49) afirman que las TIC son "el conjunto de tecnologías que permiten la adquisición, producción, almacenamiento, tratamiento, comunicación, registro, y presentación de informaciones, en forma voz, imágenes y datos, contenidas en señales de naturaleza acústica, óptica o electromagnética”. Para ello, se utilizan las fuentes de información que están divididas en voz, datos e imágenes, sin olvidar que existe otro tipo de fuentes como la información que provienen del tacto, (por ejemplo la textura de un objeto), o también aquella información que se obtiene a través de sensores.

A su vez Martínez Sánchez (1996), citado en Baelo y Cantón (2009: 102), señala que "podemos entender por nuevas tecnologías a todos aquellos medios de comunicación y de tratamiento de la información que van surgiendo de la unión de los avances propiciados por el desarrollo de la tecnología electrónica y las herramientas conceptuales, tanto conocidas como aquellas otras que vayan siendo desarrolladas como consecuencia de la utilización de estas mismas nuevas tecnologías y del avance del conocimiento humano". No obstante, Tirado (1998), hace una diferencia con la tecnología, ya que la divide en dos, "nuevas tecnologías y tecnologías avanzadas distando unas de otras por aspectos relacionados con la interactividad y la flexibilidad espacio-temporal”. De forma similar, Haag, Cummings, y McCubbrey (2004) consideran que las tecnologías de la información están compuestas de "cualquier herramienta basada en los ordenadores y que la gente utiliza para trabajar con la información, apoyar a la información y procesar las necesidades de información".

Finalmente, desde una perspectiva institucional, la OCDE (Organization for Economic Cooperation and Development) (2002: 2), señala que las TIC son "aquellos dispositivos que capturan, transmiten y despliegan datos e información electrónica y que apoyan el crecimiento y desarrollo económico de la industria manufacturera y de servicios". En conclusión, las TIC sirven en gran medida para lograr mayor crecimiento en el mercado y desarrollar nuevos productos, con mayor publicidad y, en muchos, casos a bajos costos.

\section{METODOLOGÍA}

La metodología utilizada pertenece al paradigma funcionalista planteado por Durango (2005) y tal como lo expresa Camacho (2003), en este caso, referida a una mejor descripción de las diferentes necesidades de información en las Pymes de Tundama y Sugamuxi.

\subsection{TIPO Y MÉTODO DE INVESTIGACIÓN}

El estudio fue de carácter descriptivo-explicativo, para realizar una interpretación de las variables implicadas en el objeto de investigación del presente estudio. 


\subsection{FUENTE Y TÉCNICA DE RECOLECCIÓN DE INFORMACIÓN}

Como fuentes primarias (Tamayo, 2002) se tuvieron en cuenta a los gerentes o administradores de las medianas y grandes empresas (Pymes) de Tundama y Sugamuxi. Como técnica de recolección de información se utilizó la encuesta adaptada por el investigador C. Enrique González Suárez¹. Para las fuentes secundarias (Mora y Sepúlveda, 2001), se llevó a cabo la revisión de la literatura en una primera fase.

\subsection{POBLACIÓN, MUESTRA, TIPO DE MUESTREO Y TRATAMIENTO DE LA INFORMACIÓN}

La población objeto de la investigación estuvo conformada por 266 empresas (pequeñas y medianas), distribuidas por tamaño en las principales ciudades los valles de Tundama y Sugamuxi del departamento de Boyacá, tomando como marco de referencia el censo de población de 2005. Para poder clasificar las empresas, se tuvo en cuenta la ley 1111 de 2006, que estipula un margen de Unidades de Valor Tributario (UVT), los activos totales y el número de empleados. Con respecto al sistema de muestreo, se aplicó el denominado aleatorio simple, para el tratamiento de la información, la herramienta utilizada fue el paquete SPSS versión 18, complementado con el MS. Excel.

\subsection{FICHA TÉCNICA PROPUESTA}

Como complemento al presente documento se presenta la ficha técnica del estudio.

\section{Ficha técnica tentativa del muestreo}

\begin{tabular}{|l|l|}
\hline \multicolumn{2}{|c|}{ FICHA TÉCNICA } \\
\hline Fecha de realización encuesta & Noviembre de 2013 a noviembre de 2014. \\
\hline Institución & Universidad Pedagógica y Tecnológica de Colombia. \\
\hline Facultad & Sede Sogamoso. \\
\hline Escuela & Administración de Empresas. \\
\hline Departamento & Boyacá. \\
\hline Zona de aplicación & Jurisdicción de las Cámaras de Comercio de las provincias de \\
Tundama y Sugamuxi. \\
\hline Población & Pequeñas y Medianas empresas del Departamento de Boyacá. \\
\hline Tamaño de la población & 266 empresas encuestadas. \\
\hline Procedimiento de recolección & Muestreo aleatorio simple. \\
\hline Tipo de escala de medición & Likert en las dos primeras partes del instrumento. \\
\hline Sistema de procesamiento & SPSS ${ }^{\text {TM }}$ (v. 18). \\
\hline Metodología & Encuesta. Cuestionario Escrito. \\
\hline Unidad de análisis & Gerentes y administradores. \\
\hline Realizador del estudio & Investigadores del proyecto. \\
\hline Prueba piloto & $\begin{array}{l}\text { Se aplicaron un total de } 25 \text { encuestas: } 10 \text { en Tundama y } 15 \text { en } \\
\text { Sugamuxi. }\end{array}$ \\
\hline
\end{tabular}

1. CICMA - Centro de Investigaciones de Construcción de Maquinarias. E-mail: comerca@enet.cu. 


\section{RESULTADOS DEL ESTUDIO}

El estudio inicialmente se fundamentó en un sistema de muestreo aleatorio para ambas provincias, aunque en su fase posterior, en algunos casos, se utilizó el muestreo por conveniencia, debido a que muchas de las encuestas no fueron respondidas o se perdieron por parte de los encuestados. Así, la distribución quedó establecida con 173 unidades (65\%) muéstrales para Sugamuxi y 93 (35\%) para la provincia de Tundama.

Tabla 1. Distribución muestral de estudio por provincias

\begin{tabular}{|c|l|c|c|c|c|}
\hline \multicolumn{2}{|c|}{} & Frecuencia & Porcentaje & $\begin{array}{c}\text { Porcentaje } \\
\text { válido }\end{array}$ & $\begin{array}{c}\text { Porcentaje } \\
\text { acumulado }\end{array}$ \\
\hline \multirow{3}{*}{ Válido } & Sugamuxi & 173 & 65,0 & 65,0 & 65,0 \\
\cline { 2 - 6 } & Tundama & 93 & 35,0 & 35,0 & 35,0 \\
\cline { 2 - 6 } & Total & 266 & 100,0 & 100,0 & 100,0 \\
\hline
\end{tabular}

Con relación a la distribución de las entidades por actividad económica, se logró establecer que el porcentaje predominante es el de la empresas de servicios con un $48,1 \%$, seguido de un 36,55 de las empresas comerciales, y finalmente de un 15,4 de las denominadas industriales. Este comportamiento es lógico en una región caracterizada por sectores golpeados como la agricultura y la minería, lo que ha conllevado a buscar nuevas formas de actividad empresarial, enfocándose en la prestación de servicios, por ser las de menos infraestructura para su puesta en marcha. Cabe resaltar que las más representativas fueron entidades dedicadas al equipamento tecnológico (21,42\%), seguidas del diseño e introducción de nuevos materiales $(17,8 \%)$ y procesos tecnológicos progresivos con un $17,4 \%$.

Tabla 2. Tipo de Empresa del estudio

\begin{tabular}{|c|c|c|c|c|c|}
\hline \multicolumn{2}{|c|}{} & Frecuencia & Porcentaje & $\begin{array}{c}\text { Porcentaje } \\
\text { válido }\end{array}$ & $\begin{array}{c}\text { Porcentaje } \\
\text { acumulado }\end{array}$ \\
\hline \multirow{4}{*}{ Válido } & Industriales & 41 & 15,4 & 15,4 & 15,4 \\
\cline { 2 - 6 } & Comerciales & 97 & 36,5 & 36,5 & 51,9 \\
\cline { 2 - 6 } & De servicios & 128 & 48,1 & 48,1 & 100,0 \\
\cline { 2 - 6 } & Total & $\mathbf{2 6 6}$ & $\mathbf{1 0 0 , 0}$ & $\mathbf{1 0 0 , 0}$ & \\
\hline
\end{tabular}

En cuanto al estudio, el primer interrogante fue sobre los tipos de búsqueda de información, según su calidad e intensidad. El 42,5\% de los gerentes manifestó que la información requerida es ligeramente selectiva, en tanto que 32,7\% manifestó que las necesidades informativas son muy selectivas de acuerdo con la complejidad de las labores desarrolladas al interior de la organización. 
Tabla 3. Tipos de Búsqueda de información

\begin{tabular}{|c|l|c|c|c|c|}
\hline \multicolumn{2}{|c|}{} & Frecuencia & Porcentaje & $\begin{array}{c}\text { Porcentaje } \\
\text { válido }\end{array}$ & $\begin{array}{c}\text { Porcentaje } \\
\text { acumulado }\end{array}$ \\
\hline \multirow{7}{*}{ Válido } & 0 & 4 & 1,5 & 1,5 & 1,5 \\
\cline { 2 - 6 } & Exhaustiva & 62 & 23,3 & 23,3 & 24,8 \\
\cline { 2 - 6 } & Ligeramente Selectiva & 113 & 42,5 & 42,5 & 67,3 \\
\cline { 2 - 6 } & Muy selectiva & 87 & 32,7 & 32,7 & 100,0 \\
\cline { 2 - 6 } & Total & $\mathbf{2 6 6}$ & $\mathbf{1 0 0 , 0}$ & $\mathbf{1 0 0 , 0}$ & \\
\hline
\end{tabular}

El segundo interrogante fue sobre el uso de la información, logrando establecer que 39,8\% de los administradores declaró que ésta se utiliza con el objetivo de realizar algún tipo de diagnóstico empresarial, seguida de 31,8\% para la realización de investigaciones (como es el caso de mercados), concluyendo con 12\% para otros usos como actividades recreativas, de esparcimiento, cultura general y de simple curiosidad. Uno de los usos menos frecuentes de la información son los fines académicos (artículos, ponencias o conferencias) con un 5,6\%. Esto se debe a que muy pocas empresas participan en eventos académicos e investigativos en sus áreas del conocimiento y a que la relación de instituciones de educación no superaba el 3\% de las empresas encuestadas.

Tabla 4. ¿En qué se utiliza la información?

\begin{tabular}{|c|c|c|c|c|}
\hline & \multicolumn{2}{|c|}{ Respuestas } & \multirow{2}{*}{$\begin{array}{c}\text { Porcentaje } \\
\text { de casos }\end{array}$} \\
\hline & & $\mathrm{N}$ & Porcentaje & \\
\hline \multirow{6}{*}{$\begin{array}{l}\text { La Información } \\
\text { se va a usar en }\end{array}$} & Realizar una Investigación & 109 & $31,8 \%$ & $42,4 \%$ \\
\hline & $\begin{array}{l}\text { Preparar un artículo, ponencia, } \\
\text { disertación, etc. }\end{array}$ & 19 & $5,6 \%$ & $7,4 \%$ \\
\hline & $\begin{array}{l}\text { Confeccionar un programa de } \\
\text { Investigación }\end{array}$ & 19 & $5,6 \%$ & $7,4 \%$ \\
\hline & Elaborar una reseña & 18 & $5,2 \%$ & $7,0 \%$ \\
\hline & Hacer diagnóstico & 136 & $39,8 \%$ & $52,9 \%$ \\
\hline & Otros & 41 & $12,0 \%$ & $16,0 \%$ \\
\hline \multicolumn{2}{|l|}{ Total } & 342 & $100,0 \%$ & \\
\hline
\end{tabular}

a. Grupo de dicotomía tabulado en el valor 1 .

El tercer interrogante giró en torno al contenido de la información suministrada y se encontró que la más utilizada es la descripción de materiales $(11,8 \%)$, seguida por la descripción de equipos $(11,7 \%)$, por la de procesos tecnológicos $(8,9 \%)$ y por la de las Normas $(8,4 \%)$. Este último uso parece muy evidente ya que las entidades están sujetas a todo tipo de requerimientos legales y deben estar al tanto de todas las acciones jurídicas del mundo empresarial. Otras normas como las del sistema de gestión de calidad o las de buenas prácticas en procesos o en manufactura, estarían por fuera de este contexto. 
Estudio de las necesidades de información en las pymes de Tundama y Sugamuxi

Rodríguez Díaz - Miryam Teresa, González Millán - José Javier, González M. - Oscar Ulises

Tabla 5. Contenido de la información a usar

\begin{tabular}{|c|c|c|c|c|}
\hline & \multicolumn{2}{|c|}{ Respuestas } & \multirow{2}{*}{$\begin{array}{l}\text { Porcentaje } \\
\text { de casos }\end{array}$} \\
\hline & & $\mathrm{N}$ & Porcentaje & \\
\hline \multirow{18}{*}{$\begin{array}{c}\text { Contenido de la } \\
\text { Información a } \\
\text { usar }^{\mathbf{a}}\end{array}$} & Tendencia, pronósticos & 69 & $7,2 \%$ & $26,4 \%$ \\
\hline & $\begin{array}{l}\text { Planteamiento de Problemas y proce- } \\
\text { dimientos }\end{array}$ & 73 & $7,7 \%$ & $28,0 \%$ \\
\hline & Análisis Teórico & 33 & $3,5 \%$ & $12,6 \%$ \\
\hline & Informes de Investigación & 35 & $3,7 \%$ & $13,4 \%$ \\
\hline & $\begin{array}{l}\text { Publicaciones sobre los resultados de } \\
\text { Trabajo de Investigación-desarrollo }\end{array}$ & 22 & $2,3 \%$ & $8,4 \%$ \\
\hline & Características Técnicas & 55 & $5,8 \%$ & $21,1 \%$ \\
\hline & Descripción de Procesos Tecnológicos & 85 & $8,9 \%$ & $32,6 \%$ \\
\hline & Descripción de equipos & 111 & $11,7 \%$ & $42,5 \%$ \\
\hline & Descripción de materiales & 112 & $11,8 \%$ & $42,9 \%$ \\
\hline & $\begin{array}{l}\text { Descripción de instrumentos de medi- } \\
\text { ción }\end{array}$ & 55 & $5,8 \%$ & $21,1 \%$ \\
\hline & $\begin{array}{l}\text { Descripción de instrumentos de con- } \\
\text { trol }\end{array}$ & 58 & $6,1 \%$ & $22,2 \%$ \\
\hline & Descripción de otros & 6 & $0,6 \%$ & $2,3 \%$ \\
\hline & $\begin{array}{l}\text { Métodos y medios para elevar la cali- } \\
\text { dad }\end{array}$ & 39 & $4,0 \%$ & $14,9 \%$ \\
\hline & Análisis técnico económico & 42 & $4,4 \%$ & $16,1 \%$ \\
\hline & Normas & 80 & $8,4 \%$ & $30,7 \%$ \\
\hline & Coyuntura de mercado & 43 & $4,5 \%$ & $16,5 \%$ \\
\hline & $\begin{array}{l}\text { Indicadores de volúmenes de produc- } \\
\text { ción }\end{array}$ & 31 & $3,3 \%$ & $11,9 \%$ \\
\hline & Otros & 3 & $0,3 \%$ & $1,1 \%$ \\
\hline & Total & 952 & $100,0 \%$ & \\
\hline
\end{tabular}

a. Grupo de dicotomía tabulado en el valor 1.

El cuarto interrogante fue sobre el tipo de información que se requiere, en primer lugar se encontró la aplicación de información netamente práctica con $26,3 \%$, seguida de la concerniente al tema empresarial con $20,9 \%$ y de la que refiere temas laborales con $17,1 \%$, para culminar con $16,9 \%$ de la parte técnica. Dichas respuestas tienen su lógica, en vista de las necesidades propias del sector más referenciado en el estudio. 
Tabla 6. Tipo de información

\begin{tabular}{|c|c|c|c|c|}
\hline & \multicolumn{2}{|c|}{ Respuestas } & \multirow{2}{*}{$\begin{array}{l}\text { Porcentaje de } \\
\text { casos }\end{array}$} \\
\hline & & $\mathrm{N}$ & Porcentaje & \\
\hline \multirow{7}{*}{$\begin{array}{c}\text { Tipo de } \\
\text { Información } \\
\text { que se } \\
\text { Requiere }^{\mathrm{a}}\end{array}$} & Teórica & 56 & $10,5 \%$ & $21,5 \%$ \\
\hline & Metodológica & 42 & $7,9 \%$ & $16,1 \%$ \\
\hline & De aplicación práctica & 140 & $26,3 \%$ & $53,6 \%$ \\
\hline & Empresarial & 111 & $20,9 \%$ & $42,5 \%$ \\
\hline & Técnica & 90 & $16,9 \%$ & $34,5 \%$ \\
\hline & Laboral & 91 & $17,1 \%$ & $34,9 \%$ \\
\hline & Otros & 2 & $0,4 \%$ & $0,8 \%$ \\
\hline \multicolumn{2}{|r|}{ Total } & 532 & $100,0 \%$ & \\
\hline
\end{tabular}

a. Grupo de dicotomía tabulado en el valor 1 .

El sexto interrogante correspondió al grado de actualización de la información requerida. Se estableció que $73,3 \%$ considera que ésta debe ser muy actual, mientras que $22,6 \%$ cree que la información se encuentra en un rango de 1-5 años, en términos de su vigencia. Este comportamiento es lógico para los resultados encontrados, pues en algunos temas es necesario tener lo último en términos de información. Sin embargo, existe un tipo de información como los históricos de ventas que son datos que se encuentran en fechas anteriores para observar proyecciones y comportamientos futuros.

FIGURA 2. Grado de actualización de la información

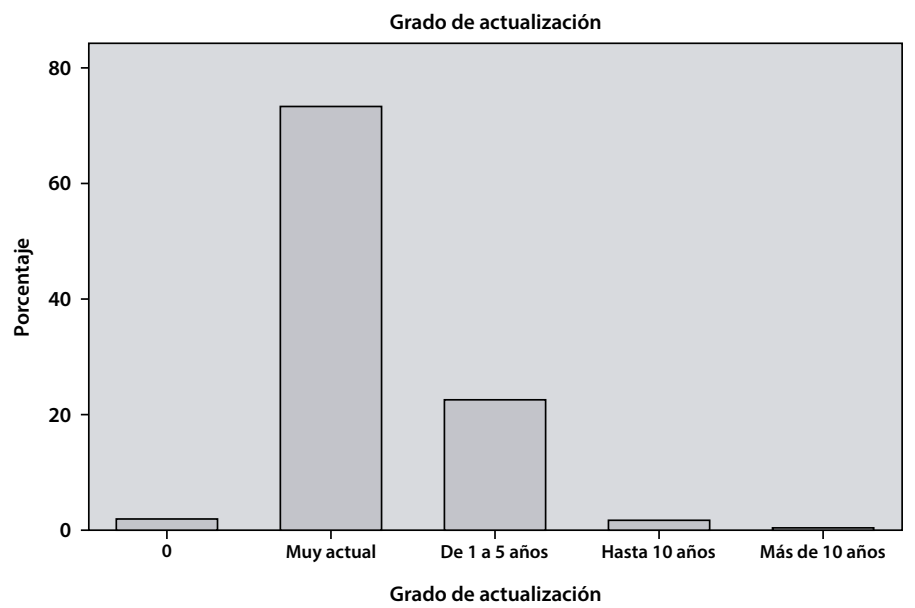

También en este punto se averiguó sobre la frecuencia de entrega de la información, en tal sentido el 54,5\% estimado en un valor de cero, demostró que es casi inmediata, lo cual se debe a la falta de procesos de planeación, seguido de un 29,4\% que corresponde a entrega diaria de información, cerrando con un 5,6\% de manera semanal. 
Estudio de las necesidades de información en las pymes de Tundama y Sugamuxi

Rodríguez Díaz - Miryam Teresa, González Millán - José Javier, González M. - Oscar Ulises

Tabla 7. Frecuencia de entrega de la información según la empresa

\begin{tabular}{|c|l|c|c|c|c|}
\hline \multicolumn{2}{|c|}{} & Frecuencia & Porcentaje & Porcentaje válido & $\begin{array}{c}\text { Porcentaje } \\
\text { acumulado }\end{array}$ \\
\hline \multirow{7}{*}{ Válido } & 0 & 145 & 54,5 & 54,5 & 54,5 \\
\cline { 2 - 6 } & Diaria & 78 & 29,4 & 29,4 & 83,9 \\
\cline { 2 - 6 } & Semanal & 15 & 5,6 & 5,6 & 89,5 \\
\cline { 2 - 6 } & Quincenal & 1 &, 4 &, 4 & 89,9 \\
\cline { 2 - 6 } & Mensual & 15 & 5,6 & 5,6 & 95,5 \\
\cline { 2 - 6 } & Bimensual & 4 & 1,5 & 1,5 & 97,0 \\
\cline { 2 - 6 } & Trimestral & 4 & 1,5 & 1,5 & 98,5 \\
\cline { 2 - 6 } & Semestral & 4 & 1,5 & 1,5 & 100,0 \\
\hline \multicolumn{7}{|c|}{} & Total & 266 & 100,0 & 100,0 & \\
\hline
\end{tabular}

En séptimo lugar se averiguó sobre las formas y medios de presentación de la información, para lo cual los gerentes manifestaron que las formas más comunes eran las de informes $(13,2 \%)$ y de texto (8,8\%). También se halló que los medios de difusión más comunes son los denominados magnéticos con un 17,3\% y los de papel con un 11,4\%. Este comportamiento es lógico dada la tendencia de presentación magnética de hoy, que supera al uso de papel, pues las empresas a pesar de ser pymes aún no entran en la ola de cero papel como concepto ecológico.

Tabla 8. Formas y Medios de presentación de Información

\begin{tabular}{|c|c|c|c|c|}
\hline & \multicolumn{2}{|c|}{ Respuestas } & \multirow{2}{*}{$\begin{array}{c}\text { Porcentaje de } \\
\text { casos }\end{array}$} \\
\hline & & $\mathrm{N}$ & Porcentaje & \\
\hline \multirow{17}{*}{$\begin{array}{c}\text { Forma de } \\
\text { presentación }\end{array}$} & Texto & 93 & $8,8 \%$ & $35,5 \%$ \\
\hline & Anotaciones & 63 & $6,0 \%$ & $24,0 \%$ \\
\hline & Resúmenes & 41 & $3,9 \%$ & $15,6 \%$ \\
\hline & Reseñas & 30 & $2,9 \%$ & $11,5 \%$ \\
\hline & Fórmulas & 20 & $1,9 \%$ & $7,6 \%$ \\
\hline & Procesos productivos & 63 & $6,0 \%$ & $24,0 \%$ \\
\hline & Informes & 139 & $13,2 \%$ & $53,1 \%$ \\
\hline & Estadísticas & 48 & $4,6 \%$ & $18,3 \%$ \\
\hline & Otros & 7 & $0,7 \%$ & $2,7 \%$ \\
\hline & Factual & 34 & $3,2 \%$ & $13,0 \%$ \\
\hline & Tablas & 84 & $8,0 \%$ & $32,1 \%$ \\
\hline & Fórmulas & 41 & $3,9 \%$ & $15,6 \%$ \\
\hline & Gráficos & 73 & $6,9 \%$ & $27,9 \%$ \\
\hline & Otros & 9 & $0,8 \%$ & $3,4 \%$ \\
\hline & Papel & 120 & $11,4 \%$ & $45,8 \%$ \\
\hline & Magnético & 182 & $17,3 \%$ & $69,5 \%$ \\
\hline & Otros & 5 & $0,5 \%$ & $1,9 \%$ \\
\hline & Total & 1052 & $100,0 \%$ & \\
\hline
\end{tabular}

a. Grupo de dicotomía tabulado en el valor 1. 
Tabla 9. Criterios que utiliza la dirección en la demanda de información

\begin{tabular}{|c|c|c|c|c|}
\hline & \multicolumn{2}{|c|}{ Respuestas } & \multirow{2}{*}{$\begin{array}{c}\text { Porcentaje de } \\
\text { casos }\end{array}$} \\
\hline & & $\mathrm{N}$ & Porcentaje & \\
\hline \multirow{9}{*}{$\begin{array}{c}\text { Criterios que utiliza la } \\
\text { direccion }^{\text {a }}\end{array}$} & Contenido temático & 77 & $21,2 \%$ & $32,0 \%$ \\
\hline & Novedad & 112 & $30,8 \%$ & $46,5 \%$ \\
\hline & Representatividad & 68 & $18,7 \%$ & $28,2 \%$ \\
\hline & Otros & 6 & $1,6 \%$ & $2,5 \%$ \\
\hline & Idiomas & 10 & $2,7 \%$ & $4,1 \%$ \\
\hline & Pluridiversidad & 24 & $6,6 \%$ & $10,0 \%$ \\
\hline & Actividad & 60 & $16,5 \%$ & $24,9 \%$ \\
\hline & Heuristicidad & 5 & $1,4 \%$ & $2,1 \%$ \\
\hline & Otros & 2 & $0,5 \%$ & $0,8 \%$ \\
\hline \multicolumn{2}{|c|}{ Total } & 364 & $100,0 \%$ & \\
\hline
\end{tabular}

a. Grupo de dicotomía tabulado en el valor 1 .

Un noveno elemento investigado correspondió a las fuentes documentales de información más utilizadas, encontrándose que son los manuales con un 14,1\%, seguidos de los informes con $14,2 \%$ y por los estudios de mercado con $12,3 \%$, lo cual constituye una explicación lógica, pues gran parte de los empresarios requiere la información práctica y aplicada.

Tabla 10. Fuentes documentales de información

\begin{tabular}{|c|c|c|c|c|}
\hline & \multicolumn{2}{|c|}{ Respuestas } & \multirow{2}{*}{$\begin{array}{c}\text { Porcentaje } \\
\text { de casos }\end{array}$} \\
\hline & & $\mathrm{N}$ & Porcentaje & \\
\hline \multirow{17}{*}{$\begin{array}{c}\text { Fuentes } \\
\text { Documentales }\end{array}$} & Libros científicos & 20 & $2,5 \%$ & $7,8 \%$ \\
\hline & Monografías & 15 & $1,9 \%$ & $5,8 \%$ \\
\hline & Libros Técnicos & 58 & $7,3 \%$ & $22,6 \%$ \\
\hline & Conferencias & 58 & $7,3 \%$ & $22,6 \%$ \\
\hline & Manuales & 112 & $14,1 \%$ & $43,6 \%$ \\
\hline & Artículos científico-técnicos & 34 & $4,3 \%$ & $13,2 \%$ \\
\hline & Materiales de eventos científicos & 11 & $1,4 \%$ & $4,3 \%$ \\
\hline & Reseñas & 20 & $2,5 \%$ & $7,8 \%$ \\
\hline & Informes & 113 & $14,2 \%$ & $44,0 \%$ \\
\hline & Documentación metodológica & 36 & $4,5 \%$ & $14,0 \%$ \\
\hline & Documentación de proyecto & 39 & $4,9 \%$ & $15,2 \%$ \\
\hline & Revistas de resúmenes & 36 & $4,5 \%$ & $14,0 \%$ \\
\hline & Descripción de invenciones & 26 & $3,3 \%$ & $10,1 \%$ \\
\hline & Documentación técnico normativa & 67 & $8,4 \%$ & $26,1 \%$ \\
\hline & Literatura técnico comercial & 48 & $6,0 \%$ & $18,7 \%$ \\
\hline & Estudios de mercado & 98 & $12,3 \%$ & $38,1 \%$ \\
\hline & Otros & 5 & $0,6 \%$ & $1,9 \%$ \\
\hline & Total & 796 & $100,0 \%$ & \\
\hline
\end{tabular}

a. Grupo de dicotomía tabulado en el valor 1 . 
La penúltima variable correspondió a los fines de la necesidad de información, hallando que el mayor es mantener actualizado el trabajo con 29,6\%, seguido de mantener actualizado el trabajo cotidiano con un 20,5\% y de mantener actualizado la especialidad con un $17,2 \%$.

Tabla 11. Fines de la Necesidad de Información

\begin{tabular}{|c|c|c|c|c|}
\hline & \multicolumn{2}{|c|}{ Respuestas } & \multirow{2}{*}{$\begin{array}{c}\text { Porcentaje } \\
\text { de casos }\end{array}$} \\
\hline & & $\mathbf{N}$ & Porcentaje & \\
\hline \multirow{9}{*}{$\begin{array}{l}\text { Necesidad } \\
\text { de la } \\
\text { Informacion }^{\text {al }}\end{array}$} & Para mantenerme actualizado en mi especialidad & 87 & $17,2 \%$ & $34,8 \%$ \\
\hline & Para mantenerme actualizado en el tema que trabajo & 150 & $29,6 \%$ & $60,0 \%$ \\
\hline & Para mantenerme actualizado en las disciplinas afines & 55 & $10,9 \%$ & $22,0 \%$ \\
\hline & Para mantenerme actualizado en mi trabajo cotidiano & 104 & $20,5 \%$ & $41,6 \%$ \\
\hline & Para comenzar un nuevo proyecto & 54 & $10,7 \%$ & $21,6 \%$ \\
\hline & Para ejecutar un proyecto de investigación científica & 14 & $2,8 \%$ & $5,6 \%$ \\
\hline & Para confeccionar un documento & 29 & $5,7 \%$ & $11,6 \%$ \\
\hline & Para trabajos docentes & 10 & $2,0 \%$ & $4,0 \%$ \\
\hline & Otros & 3 & $0,6 \%$ & $1,2 \%$ \\
\hline \multicolumn{2}{|r|}{ Total } & 506 & $100,0 \%$ & \\
\hline
\end{tabular}

a. Grupo de dicotomía tabulado en el valor 1.

Por último, en relación al tema de si el trabajo del área depende de la información de otras áreas, se encontró que 64,7\% de los casos considera que sí, mientras que $35,3 \%$ expresa que la información del área es independiente.

FIGURA 3. Del trabajo del Área con otras de la empresa

¿Depende su trabajo o parte de él, de los datos que le suministra otras áreas de trabajo?

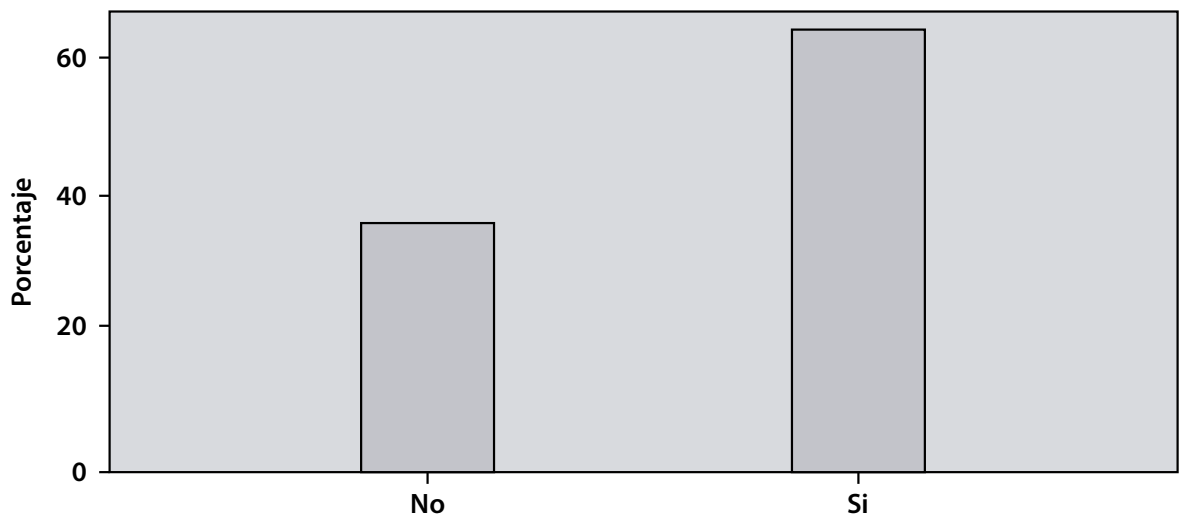




\section{CONCLUSIONES}

Se concluyó que la necesidad de información para las pymes de los valles de Tundama y Sugamuxi está relacionada principalmente con motivos empresariales y de actualización de la información.

Los distintos sistemas de información utilizados por los gerentes y/o administradores para la toma racional de decisiones se encuentran subutilizados, puesto que no llegan al mínimo aceptable del total de las herramientas tecnológicas conocidas hoy.

Es evidente que las necesidades informativas a nivel empresarial existen a todo nivel, pues se requieren en todas las dependencias de la organización. Este hecho hace que sean casi constante sus flujos de cirdulacion con diferentes fienes corporativos.

Existe una baja percepción a cerca del uso de los sistemas de información, máxime cuando se carece del uso de bases de datos o de sistemas de avanzada en relación con las herramientas de apoyo gerencial.

Dentro del torrente de necesidades de información, las relacionadas con estudios de mercados, conocimientos de manuales, normas, procedimientos y operación de equipos son las más utilizadas por las pymes de Tundama y Sugamuxi.

\section{REFERENCIAS}

1. BAELO, R., \& CANTÓN, I. (2009). Las tecnologías de la información y la comunicación en la educación superior. Estudio descriptivo y de revisión. Revista Iberoamericana de Educación / Revista Ibero-americana de Educação ISSN: 1681-5653 nº. 50/7 - 10 de noviembre de 2009.

2. BONILLA, F. M., \& CUBILLOS, D. S. (26 de febrero de 2012). Origen, Historia y Evolución de las Tics. Recuperado el 14 de abril de 2015, de: https:// sities.google.com/site/ticsyopal5/assignments

3. CABERO, J. (1998) Impacto de las nuevas tecnologías de la información y la comunicación en las organizaciones educativas. En Lorenzo, M. y otros (coords.): Enfoques en la organización y dirección de instituciones educativas formales y no formales (pp. 197-206). Granada: Grupo Editorial Universitario.

4. CAMACHO, B. (2003). Metodología de la investigación científica, un camino fácil de recorrer para todos. Tunja: Universidad Pedagógica y Tecnológica de Colombia.

5. CALVA, J. (2009). Satisfacción de Usuarios: La investigación sobre las Necesidades de la Información. Cuadernos de Investigación 11. Centro Universitario de Investigaciones Bibliotecológicas. Universidad Nacional Autónoma de México.

6. CALVA, J. (s.f). (1991). Una aproximación a lo que son las necesidades de la información. Estado de Desarrollo, 33-35. 
7. COLL, C., MAURI, M. y ONRUBIA J. (2008). Análisis de los usos reales de las TIC en contextos educativos formales: una aproximación socio-cultural. Revista electrónica de investigación educativa, 10(1), pp. 1-18. Recuperado 04/03/2014 de: http://www.scielo.org.mx/scielo. php?script=sci_arttext\&pid=S1607-40412008000100001\&lng=es\&tlng=enESTEVE.

8. DURANGO, C. (2005). Fundamentación Epistemológica de los estudios organizacionales. Medellín, Colombia: Universidad Pontificia Bolivariana.

9. FOGUET, 0., y DONÉS, F. (2011). Information and communication technologies.

10. HAAG, S., CUMMINGS, M., \& MCCUBBREY, D. J. (2004). Management information systems for the information age (4a Ed.). New York: McGraw- Hill.

11. HENAO, F. (2006). Introducción a la salud ocupacional.1ed. Bogotá, Colombia. Ecoe (Eds.).

12. LOSADA, M. (2010). Módulo de Evaluación e intervención en las condiciones de trabajo, posgrado en Salud y Seguridad en el Trabajo. Facultad de Enfermería. Universidad Nacional de Colombia. Bogotá D.C.

13. MALDONADO, G., MARTínEZ, M., GARCÍA, D., AGUILERA, L., GONZÁLEZ, M. (2010). La influencia de las TICs en el rendimiento de la PyME de Aguascalientes. Investigación y Ciencia. 47 (Abril), pp. 57-65.

14. MARTínEZ SÁNCHEZ, Francisco (1996). La enseñanza ante los nuevos canales de información, en: TEJEDOR, F. J. y GARCÍA VALCÁRCEL, A. (Eds.): Perspectivas de las nuevas tecnologías en la educación. Madrid: Narcea, pp. 101-119.

15. MARX, K. (1946). El capital: Crítica de la Economía Política, 1 edición en Español. México: Fondo de Cultura Económica.

16. MEDINA, A. (2012). Competencia de la información: Definir una necesidad de la información. Recuperado el 10 de mayo de 2015, de: http:// es.slideshare.net/crevirtualnuco/definir-una-necesidad-de-informacin-13046747.

17. MORA, M., SEPÚLVEDA, P. (2001). Metodología de la Investigación, Características del Método Científico, Balderas, México: Limusa Noriega Editores.

18. MULET, C. y PUPO, F. (2009). La Inteligencia Empresarial como función de trabajo de la administración. Contribuciones a la Economía, agosto. Recuperado el 28/01/2014 de: http://www.eumed.net/ce/2009a/.

19. OCDE (Organization for Economic Co-operation and Development) (2002). Reviewing the ICT sector definition: Issues for discussion. Recuperado el 15 de mayo de 2015, de http://www.oecd.org/dataoecd/3/8/20627293.pdf.

20. ROJAS, Y. (2004). Organización de la información: un factor determinante en la gestión empresarial. Acimed. 12 (29) (pp. 1-12). Recuperado el 28/01/2014 de: http://scielo.sld.cu/pdf/aci/v12n2/aci12204.pdf.

21. ROSARI0, J. (2006). TIC: Su uso como herramienta para el fortalecimiento y el desarrollo de la educación virtual. Archivo del Observatorio para la CiberSociedad. Recuperado el 28/01/2014 de: http://www.cibersociedad.net/archivo/articulo.php?art=221.

22. SALGUERO, A. (2009). La integración de las tecnologías de información. Hekademos, revista educativa digital, pp. 45-56.

23. SEVERIN, E. (2014). Enfoques Estratégicos sobre las TIC en Educación en América Latina y el Caribe. Chile: Acción Digital.

24. SOLER, V. (2008). El uso de las TIC (Tecnologías de la Información y la Comunicación) como herramienta didáctica en la escuela. Contribuciones a las Ciencias Sociales, octubre. Recuperado el 28/01/2014 de: www.eumed.net/rev/cccss/02/vsp.htm.

25. TAMAYO, M., (2002). El Proceso de la Investigación Científica. Limusa Noriega Editores, Balderas, México.

26. TIRADO MORUETA, Ramón (1998). Las tecnologías avanzadas en la enseñanza: aspectos psicopedagógicos, en Comunicar: Revista científica iberoamericana de comunicación y educación, núm. 10, Huelva: Grupo Comunicar, pp. 192-197.

27. VILAR, J. (s.f.). El papel de las TIC en el siglo XXI. Infonova Consultores. Recuperado el 28/01/2014 de: http://www.etuttor.com/index.php/ component/k2/item/284-not1. 